\section{IN THE NEWS}

\section{Fighting fat}

Scientists working at The

Rockefeller University in New

York City, and the Joslin

Diabetes Center in Boston,

have identified a gene that

counteracts obesity. The

gene, Foxa-2, which is

expressed in immature fat

cells (pre-adipocytes) and in

mature adipocytes of

genetically and diet-induced obese mice, prevents pre-

adipocyte differentiation and

increases metabolism in

mature adipocytes. The

findings are reported in

The Journal of Clinical

Investigation.

"We know a lot about the

various molecular pathways

that stimulate or promote fat

production, and the focus

has been on trying to block

these pathways to fight

obesity" says Markus Stoffel,

of Rockefeller University, the

lead investigator in the study.

"This pathway is one of only

a few that we know of that

naturally work to counteract

obesity" (ScienceDaily, 16 July 2003).

Overexpressing Foxa-2 in

cultured pre-adipocytes

inhibits differentiation by

activating transcription of

Pref-1. This, in turn, prevents

the adipocyte maturing. In

mature adipocytes, as well

as activating Pref-1, Foxa-2

also seemed to activate

other genes that are

involved in fat and glucose

metabolism and so might

feature in diet-induced

insulin-resistance and

benefit type 2 diabetic

patients, too.

"It is now apparent that when we overfeed a mouse and it becomes obese,

Foxa-2 is induced, which

then activates a set of genes

that work against obesity,"

says Stoffel. "Obviously

that's not sufficient to

prevent obesity, but it is

sufficient to slow it down.

Without this force, the mice

accumulate more fat."

(Yahoo News, 21 July

2003). The next challenge is

to identify what induces

Foxa-2 expression in

humans.

Kate Turner,

University of Manchester, UK

\section{PROTEIN SORTING}

\section{Over to you, SAM}

Most mitochondrial proteins are made in the cytosol, so to reach their final destination they need to be transported through the TOM (translocase of the outer mitochondrial membrane) and TIM (translocase of the inner mitochondrial membrane) complexes. In addition to protein transport, it has been thought that these translocases also sort proteins to their sub-mitochondrial destination. However, in Nature, Meisinger and colleagues now reveal that another complex - SAM functions after TOM to sort and assemble complex outer membrane proteins.

The Tom components of the TOM complex are encoded in the nucleus, so they are imported into mitochondria through TOM. The biogenesis of Tom 40 - which forms the TOM channel — requires nearly all of the TOM subunits, and Meisinger and co-workers began their work by showing that the outer mitochondrial membrane protein Mas37 is also involved in

\section{STEM CELLS}

\section{Taking new orders}

Nuclei that are transplanted from differentiated somatic cells to enucleated eggs of frogs or mammals take on the properties of the acceptor eggs. The molecular mechanisms that underlie this process of nuclear reprogramming are poorly understood, but involve new transcriptional instructions. Now, reporting in Current Biology, John Gurdon and colleagues show that a stem-cell marker is expressed in the nuclei of differentiated adult mouse and human cells after transplantation, and that this reprogramming does not require DNA replication.
Tom40 biogenesis. Previously, Mas37 had been proposed to be an import receptor and a Tom protein, but it was subsequently found to be neither.

To clarify the role of Mas37, Meisinger and colleagues studied the import of a ${ }^{35} \mathrm{~S}$-labelled precursor of Tom 40 into isolated wild-type and mas374 yeast mitochondria. They found that Tom 40 assembly into the core TOM complex occurs through Tom 40 assembly intermediates I and II in wild-type mitochondria and that, in mas37A mitochondria, the formation of all three complexes was strongly inhibited. The latter effect was also observed after incubating wild-type mitochondria with anti-Mas37 antibodies. They further showed that the level of the outer mitochondrial membrane protein porin was reduced in mas $37 \Delta$ mitochondria, whereas protein markers of the mitochondrial matrix or inner membrane were unaffected. Mas37 is therefore needed for Tom 40 biogenesis, but

An important activity of eggs is to induce replication, so to study the transcriptional reprogramming activity in eggs, Gurdon and colleagues chose Xenopus oocytes (growing egg cells), as they are nonreplicating but have a high transcriptional activity. Xenopus oocytes were injected with the nuclei of mouse fetal fibroblasts, and tested for the presence of Oct4 messenger RNA — a stem-cell marker - using reverse transcriptase (RT)-PCR. Mature, spliced Oct4 transcripts could be detected in oocytes that had been cultured at $18^{\circ} \mathrm{C}$. The level of the transcript was 5-10 times higher when nuclei were injected into the oocyte nucleus (germinal vesicle, GV) than when nuclei were deposited in the cytoplasm. To see whether the reprogramming activity of Xenopus oocytes was also effective for nuclei of adult mouse cells,

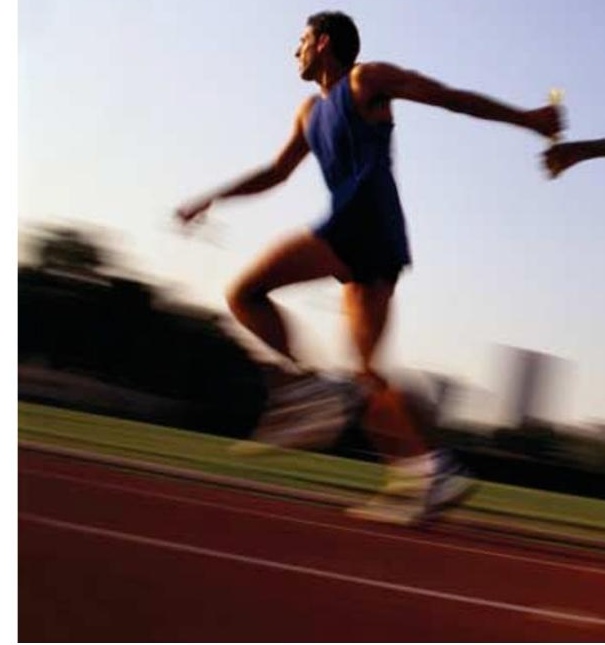

not for protein import to the inner membrane.

Next, Meisinger and co-workers studied the import and assembly of three other outer mitochondrial membrane proteins. They found that the assembly of porin and $\mathrm{Mdm} 10$ integral proteins with predicted $\beta$ strands - was strongly inhibited in mas $37 \Delta$ mitochondria, whereas the assembly of Ugo1, which has a single transmembrane segment, occurred normally. This indicates that Mas37

mouse thymocyte nuclei were injected into the GV of oocytes. Fully spliced Oct 4 transcripts could be detected in oocytes that had been incubated for less than 2 days at $18^{\circ} \mathrm{C}$. As the levels of Oct4 transcripts increased, the thymusspecific differentiation marker Thyl decreased and became undetectable by day 5.5 . Crucially, the oocyte's reprogramming activity is highly efficient, as the number of Oct4 transcripts in GVinjected oocytes after 5.5 days of incubation was comparable to that of cultured mouse embryonic-stem cells. The crossspecies reprogramming activity of Xenopus oocytes even extended to differentiated adult human cells, as the human OCT4 gene was activated in oocytes 4-6 days after GV injection with human lymphocyte nuclei.

It will be interesting to study the expression of other genes in this 